\title{
Rapid evaluation on temperature index of epoxy resin based laminated sheet using thermogravimetric point slope method
}

\author{
Liang Song,Huigang Sun \\ Corporate Research Center, ABB (China) Limited, Beijing, 100015, China
}

Keywords: Epoxy, laminated sheet, temperature index, TPS.

\begin{abstract}
The temperature index (TI) of epoxy resin based laminated sheet was studied using thermogravimetric point slope method (TPS). The main difference with standard JB/T 1544 is that a kinetic method is applied to describe the thermal gravimetric process and to calculate the activation energy. The final TI results comply with the insulation level of commercial laminated sheets under evaluation, indicating that this method can be used to study the TI of resin based laminated composites. However, its accuracy needs to be further studied.
\end{abstract}

\section{Introduction}

The thermogravimetric point slope (TPS) method was established as a standard test procedure in China (JB/T 1544 [1]) for thermal endurance characterization of impregnated varnishes and coated fabrics. The main advantage of applying this method would be a remarkable reduction in the total testing time below that required by IEC 60216 [2]. Nowadays, its use has been extended to several other kinds of insulating materials as an alternative to the standard IEC 60216. Zhang Yingsuo [3] compared the reliability of conventional and rapid ageing methods based on the theoretical analysis test data and consider that the reliability of TPS may well be identical with, or even better than conventional ageing (CA) to determine the thermal endurance properties of insulating materials. Gian etc. [4] further discussed the limits of application and the interesting characteristics of the TPS method and suggested of rearrangement of categories of insulating materials used for TPS application, which could improve the estimates and accuracy of the thermal endurance indices.

According to TPS, the whole test procedure for thermal endurance characterization shall consists of two parts: one is the realization of a thermal gravimetric (TG) test to determine the activation energy at a selected heating rate; the other is the realization of a conventional thermal endurance test at a temperature chosen in order that the time to the selected end point is large enough to ensure good accuracy for TI estimation, but not so large as in IEC 60216. That is, one TG analysis and one life test, with the total time in practice determined by the length of the life test.

In this paper, the thermal endurance characterization was performed by TG tests at three or more rates of temperature rise and analyzed by kinetic methods [5] to get the activation energy, according to the relationship as below:

$$
\frac{d \alpha}{d T}=\frac{A}{\beta} e^{-E / R T} f(\alpha)
$$

Where $\mathrm{E}$ is the activation energy, $\mathrm{R}$ is the gas constant, $\alpha$ is a temperature function of conversion and $\beta$ is the heating rate, equal to $d \mathrm{~T} / d \mathrm{t}$. The TI of epoxy resin based laminated sheet was then calculated and discussed using the TPS method.

\section{Experimental details}

\subsection{Materials}

Two kinds ofepoxy resin basedlaminated sheets reinforced by glass fiber cloth were tested and discussed. The detail information can be seen in Table 1 . 
Table 1: Materials

\begin{tabular}{ccc}
\hline Supplier & Material & Insulation level \\
\hline Shanghai Electrical Insulation & $1^{\#}$ & $\mathrm{H}$ \\
Material Co., Ltd & $2^{\#}$ & \\
Sichuan EM Technology Co., Ltd & & \\
\hline
\end{tabular}

\subsection{Thermogravimetric Analysis (TGA)}

TG measurements of fiberglass laminated sheet samples were performed using a TA Q-50 thermogravimetric analyzer at different heating rates of 5,10 and $200 \mathrm{C} / \mathrm{min}$. The laminate samples were crushed into powder then screened with 100 mesh standard sieve before test as in accordance with JB/T 1544-1999 [1].

\subsection{Mechanical Properties}

The bending strength was chosen as the diagnostic property. Sufficient samples with a size of 80 $\mathrm{mm} \times 10 \mathrm{~mm} \times 4 \mathrm{~mm}$ were put into ovens under selected ageing temperature, as shown in Fig.1a. At least 5 samples were taken out to test flexural strength at each timepoint until its value declined to $50 \%$ or even lower, as shown in Fig.1b. The bending test was performed according to GB/T 9341 [6] by Electronic universal materials testing machine (Shimadzu, AG-X) with a testing speed of 2 $\mathrm{mm} / \mathrm{min}$. The length of span between supports is $64 \mathrm{~mm}$ and the radius of the loading edge is ( $5 \pm 0.2)$ $\mathrm{mm}$. For the initial value, at least 10 specimens were tested to get an average value.
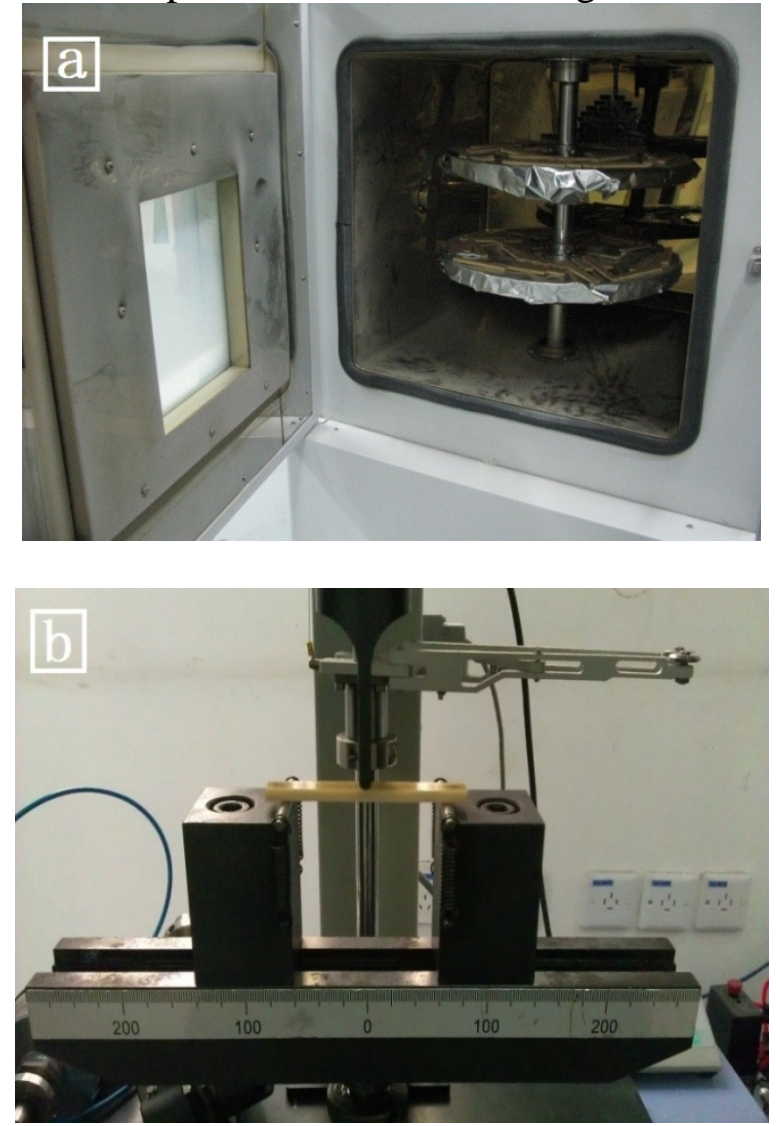

Fig.1 a) Aging oven; b) Flexural test

\section{Results and Discussion}

\subsection{Apparent Active Energy (E).}

The Kissinger method [7], which is based on the following equation was applied to calculate the apparent active energy:

$$
\ln \left(\beta / T_{\max }{ }^{2}\right)=\ln (A R / E)-E /\left(R T_{\max }\right)(2)
$$


Where $\beta$ is the heating rate, $T_{\max }$ is the temperature corresponding to the inflection point of the thermodegradation curves that corresponds to the maximum reaction rate (maximum temperature on differential TGA curves (DTG)), A is the pre-exponential factor of the Arrhenius equation, $\mathrm{R}$ is the universal gas constant, and $\mathrm{E}$ is the activation energy. This equation holds true for reactions for which the effective order is close to 1 and allows for a calculation of the activation energy that is based on the maximum temperature versus the heating rate. The plot of $\ln \left(\beta / T_{\max }{ }^{2}\right)$ versus $1 / T_{\max }$ can be fitted to a straight line, and the activation energy (E) can be calculated from the slope of the line. Fig.2 and Fig.3 show the plots of the DTG data for both $1^{\#}$ and $2^{\#}$ materials using Kissinger method.

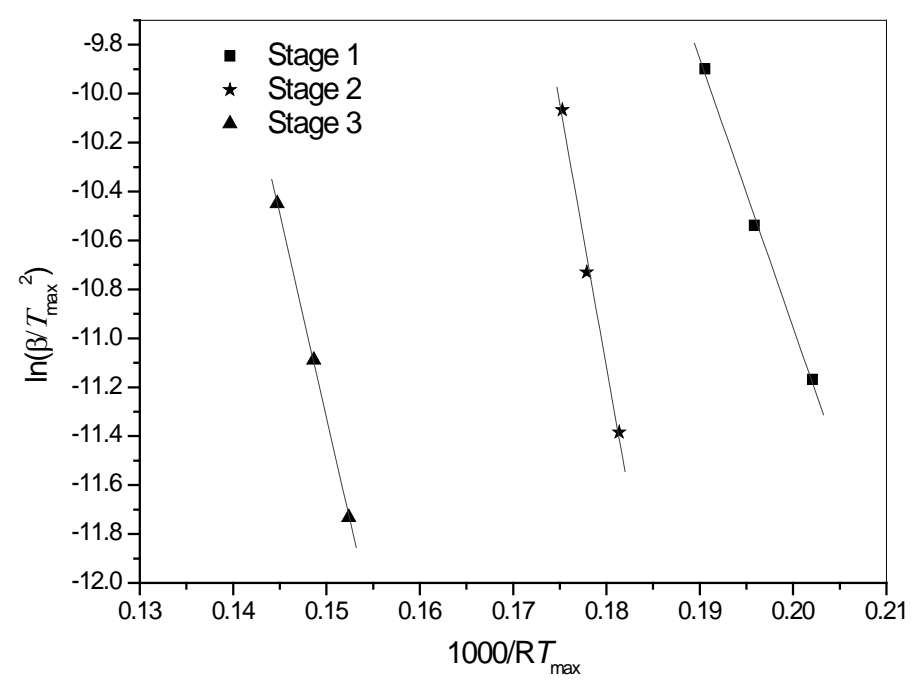

Fig.2 Dependence of the maximum temperature of DTG curves on the heating rate for $1^{\#}$ material at various stages of thermal degradation using the Kissinger equation

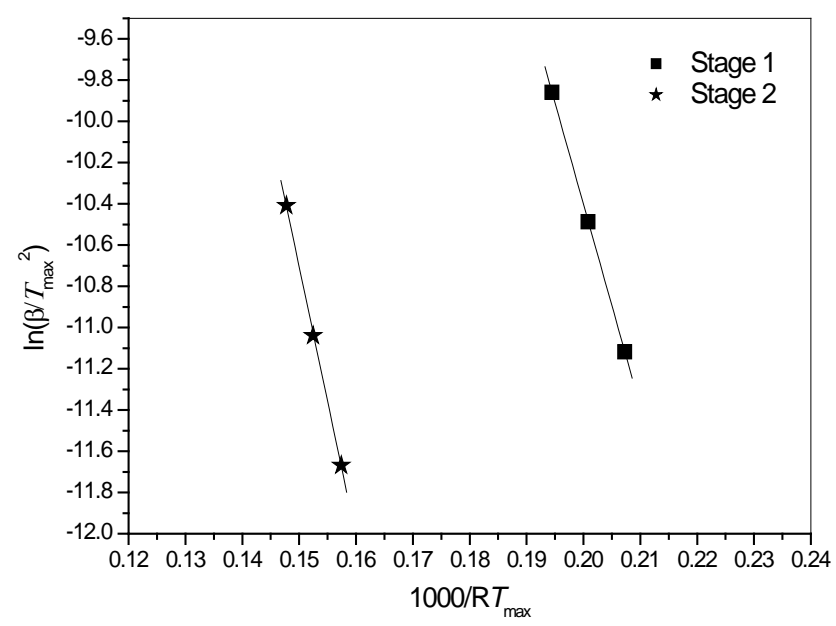

Fig.3 Dependence of the maximum temperature of DTG curves on the heating rate for $2^{\#}$ material at various stages of thermal degradation using the Kissinger equation

The advantage of this Kissinger method is that it does not require previous knowledge of the reaction mechanism for determining the activation energy. Based on above plots, the activation energy value for 1\# and 2\#materials were calculated and listed in Table 2 and Table 3, respectively. To be noted that the activation energy adopted for calculating lifetime should be corresponding to the higher conversion stage [5]. 
Table.2: Parameters of thermal degradation of $1^{\#}$ material

\begin{tabular}{|c|c|c|c|c|c|c|c|c|}
\hline Parameter & \multicolumn{3}{|c|}{ Stage 1} & \multicolumn{3}{|c|}{ Stage 2} & \multicolumn{2}{|r|}{ Stage 3} \\
\hline $\begin{array}{c}\text { Heating rate } \\
{\left[{ }^{\circ} \mathrm{C} / \mathrm{min}\right]}\end{array}$ & 5 & 10 & 20 & 5 & 10 & 20 & 5 & 10 \\
\hline $\mathrm{T}_{\max }\left[{ }^{\circ} \mathrm{C}\right]$ & 322 & 390 & 516 & 341 & 403 & 536 & 358 & $413 \quad 558$ \\
\hline Conversion & \multicolumn{3}{|c|}{$0 \sim 0.07$} & \multicolumn{3}{|c|}{$0.07 \sim 0.20$} & \multicolumn{2}{|c|}{$0.20 \sim 0.39$} \\
\hline $\mathrm{E}[\mathrm{KJ} / \mathrm{mol}]$ & \multirow{2}{*}{\multicolumn{3}{|c|}{$\begin{array}{c}109.79 \\
5734\end{array}$}} & \multicolumn{3}{|c|}{215.17} & \multicolumn{2}{|c|}{166.50} \\
\hline $\mathrm{b}$ & & & & \multicolumn{3}{|c|}{11238} & \multicolumn{2}{|r|}{8696} \\
\hline
\end{tabular}

Table 3: Parameters of thermal degradation of $2^{\#}$ material

\begin{tabular}{ccccccc}
\hline Parameter & & Stage 1 & & \multicolumn{3}{c}{ Stage 2 } \\
\hline Heating rate $\left[{ }^{\circ} \mathrm{C} / \mathrm{min}\right]$ & 5 & 10 & 20 & 5 & 10 & 20 \\
$\mathrm{~T}_{\max }\left[{ }^{\circ} \mathrm{C}\right]$ & 307 & 326 & 345 & 491 & 516 & 541 \\
Conversion & & $0 \sim 0.07$ & & $0.08 \sim 0.17$ \\
$\mathrm{E}[\mathrm{KJ} / \mathrm{mol}]$ & & 98.30 & & \multicolumn{2}{c}{130.75} \\
$\mathrm{~b}$ & & 5134 & & & 6829 & \\
\hline
\end{tabular}

\subsection{Accelerated ageing test.}

To obtain the intercept $a$ in the thermal ageing lifetime estimation equation $\lg \tau=a+b / T$, thermal accelerated ageing test of $1 \#$ and $2 \#$ materials were performed at $230 \mathrm{oC}$ and $240 \mathrm{oC}$ respectively, referring to the GB/T 11026.1 [8] recommendation and their insulation class level. And the flexural strength was taken as the property directly related to thermal oxidative reaction rate. Table 4 shows the variation of flexural strength of $1 \#$ and $2 \#$ with ageing time.

Table 4: Flexural strength of $1^{\#}$ and $2^{\#}$ materials with ageing times

\begin{tabular}{|c|c|c|c|c|c|}
\hline \multicolumn{3}{|c|}{$1^{\#}$ Material } & \multicolumn{3}{|c|}{$2^{\#}$ Material } \\
\hline $\begin{array}{c}\text { Ageing } \\
\text { time } \\
{[\mathrm{h}]}\end{array}$ & $\begin{array}{l}\text { Max. } \\
\text { Stress } \\
{[\mathrm{MPa}]}\end{array}$ & $\begin{array}{c}\text { Fraction of } \\
\text { initial value, } \\
P[\%]\end{array}$ & $\begin{array}{c}\text { Ageing } \\
\text { time } \\
{[\mathrm{h}]}\end{array}$ & $\begin{array}{l}\text { Max. } \\
\text { Stress } \\
{[\mathrm{MPa}]}\end{array}$ & $\begin{array}{c}\text { Fraction of } \\
\text { initial value, } \\
P[\%]\end{array}$ \\
\hline 0 & 436 & 100.00 & 0 & 589 & 100.00 \\
\hline 144 & 465 & 106.65 & 72 & 591 & 100.42 \\
\hline 216 & 450 & 103.21 & 240 & 549 & 93.17 \\
\hline 288 & 421 & 96.56 & 408 & 577 & 97.93 \\
\hline 360 & 379 & 86.93 & 600 & 509 & 86.40 \\
\hline 456 & 260 & 59.63 & 744 & 460 & 78.03 \\
\hline 504 & 223 & 51.15 & 936 & 344 & 58.37 \\
\hline 528 & 152 & 34.86 & 1104 & 253 & 42.92 \\
\hline
\end{tabular}

After plotting fraction of initial flexural strength against ageing time and fitting with certain mathematics methods as shown in Fig.4, from which the corresponding ageing time to get the end point (50\%) can be obtained. Then the intercept a can be further calculated. For 1\# sample, when $\mathrm{P}=50, \tau(230 \mathrm{oC})$ is $494 \mathrm{~h}$. So $\mathrm{a}=\lg [\tau(230 \mathrm{oC})]-[\mathrm{b} /(273+230)]=-14.594$. Similar for $2 \#$ sample, $\tau$ $(230 \mathrm{oC})$ is $1020 \mathrm{~h}$ when $\mathrm{P}$ reach to $50 \%$. Then a can be calculated as -10.30 . 

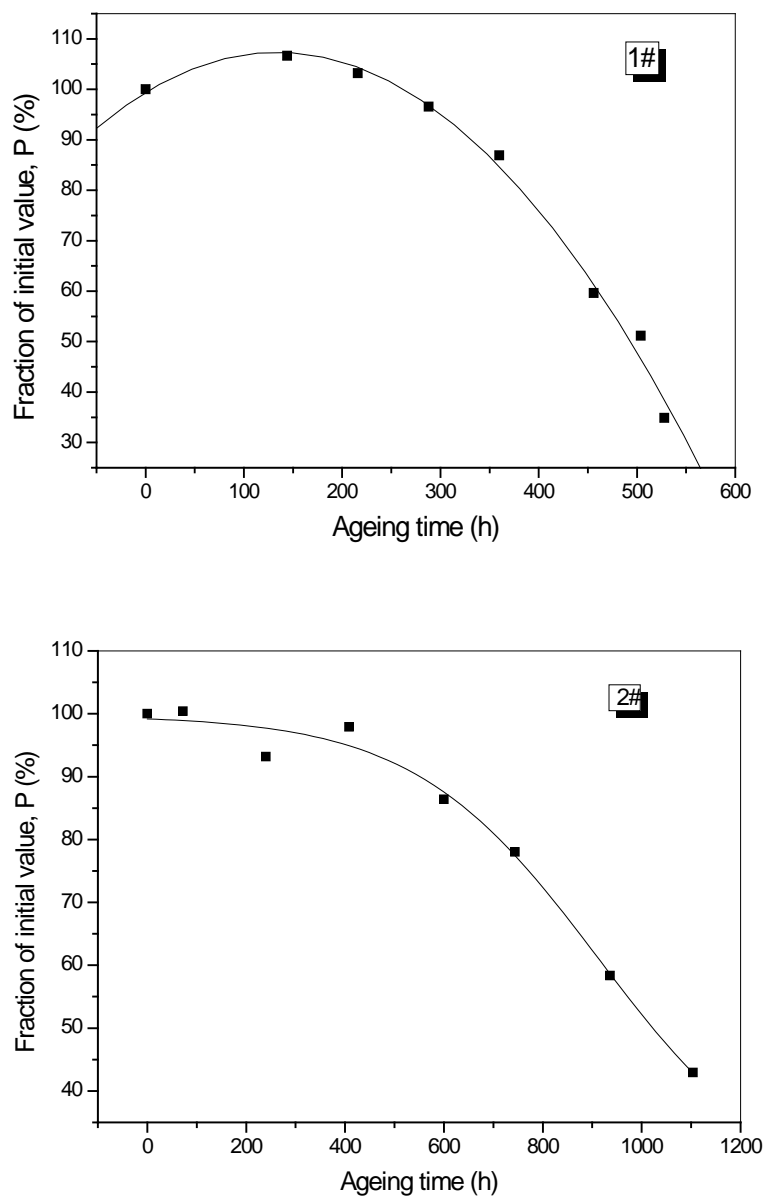

Fig.4 Plotting of fraction of initial flexural strength against ageing time for $1^{\#}$ and $2^{\#}$ materials, respectively

Therefore, the lifetime equation for $1 \#$ and $2 \#$ samples are expressed as $\lg \tau=-14.59+8696 / \mathrm{T}$ and $\lg \tau=-10.30+6829 / \mathrm{T}$, respectively. For a time of $20000 \mathrm{~h}$, the temperature index for $1 \#$ and $2 \#$ samples can be calculated based on the obtained lifetime equation. The final TI value for $1 \#$ and $2 \#$ samples are $187 \mathrm{oC}$ and $195 \mathrm{oC}$, indicating that both laminated sheets have a limiting temperature of thermal class $\mathrm{H}$ (withstanding a temperature of up to $180 \mathrm{oC}$ ).

\section{Summary}

In this paper, the temperature index of two commercial epoxy resin based laminated sheets were investigated using TPS method. A slightly different with JB/T 1544-1999 is that, the Kissinger method was introduced in calculating the activation energy. The calculated TI results are in consistence with corresponding insulation class of tested laminated sheets. It appears that this method can be used to obtain the TI value of this kind of material. However, its potential influences and accuracy still need further study.

\section{References}

[1] JB/T 1544, Method of Rapid Evaluation of Thermal Endurance Properties for Electrical Insulating Impregnated Varnishes and Coated Fabrics-Thermogravimetric Point Slope Method (TPS).

[2] IEC 60216, Guidelines for the Determination of Thermal Endurance Properties of Electrical Insulating Materials, 2001. 
[3] Zhang, Y. S,Bai Y. and Ma,Y.X,Comparison of reliability of conventional and rapid aging methods for insulating materials. IEEETransactions on Dielectrics and Electrical Insulation, 27(6), pp. 1159-1165, 1992.

[4] Gian,C.M. and Zhang,Y.S,Notes on the thermogravimetric point slope procedure for thermal endurance characterization of insulating materials. IEEE Transactions on Dielectrics and Electrical Insulation. 1(6), pp. 1026-1033, 1994.

[5] Fraga,F. and NunezE. R, Activation Energies for the epoxy system badge $n=0 / m-X D A$ obtained using data from thermogravimetric analysis.Journal of Applied Polymer Science. 80, pp. 776782, 2001.

[6] GB/T 9341, Plastic-Determination of flexural properties. In Chinese, 2008.

[7] KissingerH. E, Reaction kinetics in differential thermal analysis.Analytical Chemistry. 29, pp. 1702-1706, 1957.

[8] GB/T 11026.1, Electrical insulating materials-Properties of thermal endurance-Part 1: Ageing procedures and evaluation of test results. In Chinese, 2003. 